\title{
Enseignement bilingue et politique linguistique
}

\section{Guy Trézeux}

\section{(2) OpenEdition}

Journals

Édition électronique

URL : http://journals.openedition.org/ries/3972

DOI : $10.4000 /$ ries.3972

ISSN : 2261-4265

\section{Éditeur}

Centre international d'études pédagogiques

\section{Édition imprimée}

Date de publication : 7 septembre 1995

Pagination : 103-117

ISSN : 1254-4590

\section{Référence électronique}

Guy Trézeux, «Enseignement bilingue et politique linguistique », Revue internationale d'éducation de Sèvres [En ligne], 07 | 1995, mis en ligne le 03 octobre 2014, consulté le 19 avril 2019. URL : http:// journals.openedition.org/ries/3972 ; DOI : 10.4000/ries.3972

Ce document a été généré automatiquement le 19 avril 2019

(c) Tous droits réservés 


\title{
Enseignement bilingue et politique linguistique
}

\author{
Guy Trézeux
}

1 Depuis quelque temps en France, les articles et publications sur l'enseignement bilingue semblent se multiplier. C'est sans doute la preuve d'un intérêt nouveau pour ce type d'enseignement relativement peu répandu dans un pays à forte tradition éducative monolingue. Les nombreuses tentatives passées, en effet, n'ont reçu le plus souvent qu'une attention marginale de la part d'un grand public pourtant sensible habituellement aux problèmes éducatifs. C'est que la France n'est pas un pays spontanément ouvert au bilinguisme, et ce, en partie pour des raisons historiques. La République française a eu longtemps besoin pour ses enfants d'une langue unique, assurant l'unité de la nation et essayant de faire oublier progressivement des siècles de pratique de langues régionales ou locales, de dialectes et de patois, dont il reste peu de choses aujourd'hui, d'autant que les médias ont contribué largement à imposer une norme linguistique. À notre époque encore, la conception française de l'égalité républicaine, qui suppose l'intégration à terme des populations immigrées à la communauté nationale, risque d'aboutir à l'abandon progressif de la pratique des langues d'origine de ces populations, même si des dispositifs spécifiques sont mis en place pour qu'elles continuent à être étudiées.

2 Si un intérêt tardif se manifeste à présent pour l'enseignement bilingue alors que d'autres pays ont pu l'expérimenter à plus ou moins grande échelle, c'est sans doute sous la pression d'un monde extérieur où le français ne suffit plus pour se faire comprendre, où l'anglais joue progressivement le rôle d'une langue de communication internationale, où l'Europe devient une réalité, où les voyages représentent une nécessité, etc. Les raisons sont connues depuis longtemps et c'est sans doute leur accumulation de nos jours, leur caractère pressant aussi, qui expliquent ce renouveau d'intérêt qui ne touche toutefois pas seulement les langues étrangères mais aussi les langues régionales comme le rappelle une récente circulaire du ministère de l'Éducation. D'autre part, ce regain d'intérêt ne concerne pas que l'enseignement bilingue en France mais ce qu'on pourrait appeler l'«enseignement bilingue francophone" à l'étranger, qui sera l'objet central de cet article. 


\section{Une tendance nouvelle}

3 Entendons par enseignement bilingue "francophone" un enseignement qui utilise comme langue d'apprentissage le français parallèlement à une autre langue, généralement mais pas exclusivement la langue maternelle des élèves. Ce type d'enseignement n'est pas nouveau puisque de nombreux établissements scolaires, au Moyen-Orient notamment, le pratiquent depuis des dizaines d'années, parfois même pour certains établissements de Turquie ou d'Égypte depuis le siècle dernier. Ce qui est nouveau c'est, d'une part l'apparition récente d'un enseignement bilingue dans des pays où il n'existait pas, et d'autre part son renforcement dans des pays où il avait tendance à s'essouffler.

4 L'explication de cette tendance nouvelle est d'abord politique. Certains pays qui n'avaient pas défini de politique linguistique précise ont été amenés à en mettre en place sous la pression de faits nouveaux ; certains autres qui en avaient une ont décidé d'en changer ; enfin la France elle-même, qui mène depuis longtemps une politique linguistique extérieure déterminée, a modifié en partie sa stratégie en accordant une importance plus grande à l'enseignement bilingue francophone.

5 L'exemple des pays d'Europe centrale et orientale est significatif. Tant qu'ils se trouvaient situés dans la zone d'influence de l'ancienne URSS, le russe occupait une place évidemment prépondérante. Dès que le communisme soviétique a été remis en cause, certains de ces pays se sont tournés vers l'Europe de l'Ouest en vue d'une intégration future à l'Union européenne. Très rapidement, l'enseignement du russe a diminué et l'enseignement des langues d'Europe occidentale a augmenté, l'anglais en premier lieu, mais aussi l'allemand dans les pays de vieille influence germanique, et le français dans ceux où cette langue était déjà bien implantée (en particulier en Bulgarie où les premières sections bilingues datent de 1950, et en Roumanie). Cette option nouvelle ne s'est pas réduite au simple enseignement-apprentissage des langues concernées, mais a visé l'enseignement de disciplines scolaires diverses dans ces langues, devenues ainsi langues d'enseignement. Il semble avéré en effet que l'enseignement bilingue, qui fait de la langue un outil d'apprentissage et place l'élève dans une situation authentique d'utilisation de cette langue outil, permet l'acquisition d'une maîtrise linguistique de bien meilleure qualité que celle qui résulte d'un simple apprentissage de la langue cible.

6 C'est cette maîtrise de bon niveau qui est recherchée dans de nombreux pays désirant qu'émerge à terme, en leur sein, une espèce d'élite susceptible de communiquer facilement avec des interlocuteurs étrangers et de mettre ainsi en place des coopérations fructueuses propres à favoriser les échanges et le développement économique. La communication n'est en général pas le seul objectif recherché. L'enseignement bilingue francophone par exemple est associé souvent à la réputation de qualité du système éducatif français, même s'il s'appuie sur des programmes nationaux et est encadré par des enseignants nationaux. On pense, en effet, que la France peut et doit aider l'enseignement bilingue puisqu'il va dans le sens de ses intérêts. Par cette aide, on espère que des enseignants, des méthodes, des manuels français donneront aux cursus bilingues une orientation plus spécifiquement française.

7 "On », ça peut être des autorités locales, des chefs d'établissement, mais aussi des parents. Au Vietnam, par exemple, ce sont d'abord les familles qui ont poussé à la 
création de classes bilingues, bientôt suivies par les chefs d'établissement puis, avec des nuances, voire parfois des réticences au début, par les autorités locales et nationales.

Dans beaucoup de pays, au Moyen Orient en particulier, l'enseignement bilingue francophone est d'abord synonyme d'ouverture au monde. Dans des contextes nationaux où la religion tend à pousser à un repli culturel, on peut comprendre l'enjeu que représente l'éducation en général, et en particulier l'enseignement des langues et des cultures étrangères, et donc l'enseignement bilingue.

9 Les raisons du développement de l'enseignement bilingue dans le monde, et notamment de l'enseignement bilingue francophone, sont donc multiples et prennent souvent leurs racines dans une situation politique et économique qui, à certains moments de l'histoire des peuples, pose le problème éducatif sous la forme d'une alternative entre ouverture aux autres et repli sur soi-même. Il convient cependant de rappeler qu'une condition est première : que ces «autres » dont il est question soient perçus comme non dangereux pour l'identité culturelle des peuples qui recherchent cette ouverture. Lorsque, par exemple, un passé colonial est encore trop présent dans les mémoires, le retour de la langue du colonisateur est rarement bien venu, on s'en doute, sauf si elle peut servir de langue véhiculaire permettant d'éviter la prééminence d'une langue vernaculaire sur les autres, en Afrique par exemple.

10 L'enseignement bilingue est généralement le résultat d'un choix politique de la part du pays où il est dispensé; c'est pourquoi il a le plus souvent été mis en place par les autorités de ce pays, soit directement, soit, on l'a vu, sous la pression de familles particulièrement motivées. Il est rare que le choix de l'implantation d'un tel enseignement vienne du pays « exportateur » de sa langue, la France dans le cas qui nous intéresse. Dans cette hypothèse, en Pologne par exemple où il a été introduit en 1990 à la suite de la visite officielle d'un ministre français, une négociation est de toute façon nécessaire à plus ou moins long terme avec le pays « importateur " pour donner un statut à ce type d'enseignement lorsqu'il n'existe pas encore et dans tous les cas pour réfléchir aux contenus et aux validations.

11 L'objet de cet article est d'essayer de présenter quelques-uns des problèmes que pose l'enseignement bilingue, tant pour le pays qui l'accueille que pour celui qui, comme la France, en a fait une priorité de sa politique linguistique. On ne considérera cependant de la politique linguistique de la France que son volet extérieur, pour des raisons de facilité, mais il conviendrait, pour être complet, de réfléchir dans un autre cadre à l'articulation entre politique linguistique extérieure et politique linguistique intérieure. Si la politique linguistique intérieure de la France qui relève directement du ministère de l'éducation nationale ne peut que prendre en compte la place des différentes langues présentes sur le sol français (français, langues régionales, langues de l'immigration, langues étrangères), la politique linguistique extérieure qui relève, elle, du ministère des affaires étrangères pour la majorité des pays du monde, et du ministère de la coopération pour les pays d'Afrique francophone en particulier, n'intègre pas dans sa visée d'autres langues que le français. Elle a donc d'abord pour objet d'exporter la langue officielle unique de l'État. Autant dire que parler d'enseignement bilingue dans ce cadre, ce n'est parler que de l'enseignement du français et d'une autre langue, généralement la langue principale du pays où s'exerce cet enseignement. 


\section{Établissement bilingue et environnement linguistique}

12 L'enseignement bilingue francophone est présent sur tous les continents, en particulier dans les pays appartenant à la francophonie, et notamment en Afrique. Je me contenterai ici cependant de ne prendre des exemples que dans des zones où l'environnement francophone est faible, voire inexistant, et où l'enseignement bilingue ne s'impose donc pas d'emblée, où il est le résultat d'un choix local, donc d'une option délibérée, parfois même d'un combat militant.

Il faut noter par ailleurs que l'environnement francophone dans un pays n'est pas directement lié à l'appartenance officielle de ce pays à la communauté francophone. Il convient en effet de remarquer que coexistent deux francophonies: une francophonie linguistique, qui correspond à un véritable "partage » de la langue française et qui concerne les pays où le français est réellement langue maternelle ou langue seconde ; une francophonie géopolitique qui relève en partie du dialogue Nord-Sud et qui rassemble des pays souvent bien peu francophones au sens linguistique du terme, mais qui trouvent au sein d'un groupe de près de cinquante pays des intérêts politico-économico-culturels communs. C'est ainsi que le Vietnam, désireux de trouver la légitimité internationale qui lui manque, a présenté sa candidature pour accueillir le prochain sommet des chefs d'États francophones en 1997, sans pour autant développer un environnement linguistique qui permettrait au français d'occuper une place privilégiée dans le pays.

La place de l'enseignement bilingue dans un établissement est essentielle en ce qu'elle va le plus souvent signer l'identité de cet établissement et définir sa vocation. Elle est essentielle aussi en ce qu'elle détermine le statut de la langue dans cet établissement. Il est à noter que ce statut ne recouvre que rarement le statut officiel de la langue dans le pays. Le cas du Liban reste une exception, puisque le français y possède un statut privilégié dans la majorité des établissements scolaires, statut qui ne correspond pas à sa place véritable dans le pays, mais est le reflet de son statut officiel au sein du système éducatif. Dans de nombreux pays ayant adhéré à la francophonie et où le français peut n'avoir aucun statut officiel, les établissements bilingues offrent au français un statut généralement plus privilégié que son statut au sein du système éducatif local. Dans ce cas, l'écart qui existe entre le statut du français dans l'établissement et son statut réel à l'extérieur constitue un paramètre essentiel : l'environnement linguistique doit ainsi être pris en compte selon qu'il est, soit essentiellement, soit majoritairement, soit minoritairement francophone, ou pas francophone du tout.

Un deuxième écart est également à considérer, celui qui existe entre le type de relations que l'élève entretient avec la langue cible (autrement dit le statut personnel de cette langue pour l'individu) et le statut officiel de cette langue, dans l'établissement d'une part, dans le pays d'autre part. Pour l'élève qui débute un apprentissage dans une langue non maternelle, cette langue peut être seconde (s'il a déjà été mis en contact avec elle de manière intense avant son entrée dans l'établissement) ou étrangère. Mais dès que l'apprentissage est lancé, c'est-à-dire dès qu'un enseignement est donné dans la langue outil, on ne peut plus parler de langue étrangère au sens strict puisque la place de cette langue est celle d'une langue seconde dans l'établissement. Elle devient pour l'élève partiellement étrangère puis réellement seconde au fur et à mesure qu'elle est de plus en plus un moyen pour apprendre et de moins en moins un objet d'apprentissage (même si elle continue à être apprise par son utilisation même). Le rapport à la langue évoluant 
ainsi détermine par là-même un rapport à la culture que cette langue véhicule, rapport qui peut être positif ou négatif selon les conditions d'enseignement, les méthodes employées, les représentations individuelles ou l'image sociale de la langue concernée. Notons au passage que l'on parlera plutôt, pour suivre Hamers et Blanc, de « bilingualité » dès lors qu'il s'agit de définir et de mesurer la compétence bilingue d'un individu. Le terme «bilinguisme" sera au contraire réservé à des situations susceptibles d'être décrites d'un point de vue sociolinguistique, et qui touchent donc des groupes, des communautés, des pays.

16 La prise en compte de ces trois paramètres que sont le lieu de l'enseignement bilingue (classe, section, établissement), l'environnement linguistique extérieur et l'élève en tant qu'apprenant, et des rapports qu'ils entretiennent les uns avec les autres est première dans tout descriptif de situations scolaires bilingues et est essentielle pour toute décision éventuelle visant à la mise en place de cursus de ce type.

\section{Programmes français ou nationaux?}

17 Il faut tout d'abord noter que les frontières entre établissements dits français et sections et établissements dits bilingues ne sont pas si claires qu'il pourrait le sembler au premier abord. Certains établissements évoluent. Ainsi, tel établissement qui suivait les programmes français peut décider d'adopter les programmes nationaux, et ce pour diverses raisons, en particulier si le nombre d'élèves français est faible. Tel a été le cas il y a quelques années pour le lycée de Curico, au Chili, par exemple. La tentation contraire est fréquente: certains établissements du Moyen-Orient, du Liban en particulier, sont désireux de suivre les programmes français du fait par exemple de la trop grande ancienneté des programmes libanais, non révisés depuis vingt ans à cause de la guerre. Dans certains cas, on peut arriver à la mise en place de programmes intégrés, comme au lycée français de Montevideo. Cette dernière solution pose un problème politique: l'accord des autorités locales à de tels programmes nécessite le plus souvent une adaptation de la législation qui ne va pas de soi.

Même lorsque les programmes suivis par l'établissement sont ceux du ministère français de l'éducation nationale, il est rare qu'ils soient suivis intégralement, simplement parce que la loi locale accepte difficilement que les ressortissants du pays ne reçoivent aucun enseignement conforme aux programmes nationaux. Dans ce cas, on compose plus ou moins, selon le degré d'exigence des partenaires. Il est parfois élevé, comme au Venezuela où deux sections coexistent, une section vénézuélienne ne scolarisant que des Vénézuéliens sur la base des programmes vénézuéliens et où le français est enseigné comme langue étrangère, et une section française scolarisant en français des élèves français ou de nationalité tierce. Il est parfois faible et on est alors proche des programmes français. Il est le plus souvent intermédiaire, et l'on peut arriver à une répartition égalitaire des programmes (50/50, comme en Argentine ou en Équateur), ou à un partage donnant en général l'avantage aux programmes français pour les disciplines principales (disciplines scientifiques en particulier), de nombreuses disciplines restant enseignées selon les programmes locaux et dans la langue locale (langues, histoiregéographie, disciplines artistiques, éducation physique et sportive). Nous sommes bien alors dans le cas d'établissements bilingues et biculturels de facto, d'autant que la langue nationale reste dans tous les cas la plus parlée dans la cour de récréation, parfois même dans la classe. 
19 Si la frontière n'est pas nette pour les programmes, elle ne l'est par conséquent pas non plus pour les cursus ni pour les examens préparés. En Amérique latine, par exemple, tout établissement dit «français » s'engage à préparer ses élèves aux examens français. Il ne peut ignorer cependant que, presque toujours, ses élèves sont dans leur grande majorité destinés à suivre des études sur place, et qu'en l'occurrence le baccalauréat français ne leur servira pas à grand chose. C'est pourquoi la plupart des établissements scolaires «français» permettent à leurs grands élèves de préparer l'examen de fin d'études secondaires et (ou) les examens d'entrée à l'université. Cette préparation peut être parallèle à la préparation du baccalauréat français, ce qui alourdit considérablement la charge horaire des élèves, ou se faire séparément. Dans ce dernier cas, en particulier quand l'entrée à l'université locale se fait avant la fin des études secondaires françaises, à Lima par exemple, l'établissement peut posséder une section spécifique d'enseignement national en fin de cursus destiné aux élèves ne désirant pas passer le baccalauréat français, et ce malgré une scolarité majoritairement francophone.

Inversement, certains établissements à cursus bilingues sont tentés de franchir la frontière qui les sépare des établissements français et de préparer leurs élèves au baccalauréat. Il en a été question il y a quelques années en Bulgarie par exemple. Les raisons invoquées peuvent sembler légitimes : les programmes français paraissent dans certains pays plus audacieux que les programmes nationaux ou plus proches des acquis récents des différentes didactiques; le baccalauréat français a une bonne image de marque internationale et, à ce titre, sa préparation peut dans certains pays contribuer à la réputation des établissements où il est préparé (Liban et Égypte notamment); il permet d'accéder aux universités françaises, particulièrement bon marché si on les compare aux universités des autres pays occidentaux, et qui accueillent déjà cent cinquante mille étudiants étrangers, plaçant ainsi la France au deuxième rang des pays d'accueil pour les études supérieures après les États-Unis. C'est lui également qui garantit une éducation « à la française ", sans laquelle l'enseignement bilingue risque de se réduire à un simple apprentissage linguistique; beaucoup s'accordent en effet, à tort ou à raison, à reconnaître que l'enseignement français, plus que d'autres, donnerait priorité à l'expression écrite et à la réflexion critique. Ceci est particulièrement net dans les examens qui imposent des épreuves rédigées, au contraire des QCM anglo-saxons. De plus, la préparation du baccalauréat permet de résoudre le sempiternel problème qui se pose partout où existe un enseignement bilingue : celui des manuels, jamais adaptés ou rarement, à un type d'enseignement qui reste toujours très minoritaire. Il suffit alors dans ce cas d'utiliser les manuels français dont le coût cependant reste élevé pour nombre de pays.

21 Les arguments qui s'opposent à la préparation du baccalauréat dans ce type d'établissements sont également fort recevables. Le premier problème est celui de la formation des enseignants locaux, non seulement au plan linguistique, puisqu'ils doivent évidemment posséder un excellent niveau de français, mais aussi aux plans pédagogique et surtout didactique puisqu'il s'agit alors d'enseigner des programmes différents de ceux des pays d'origine de ces enseignants. Le problème de l'existence ou non d'un centre d'examen local n'est pas moindre. La mise en place d'un tel centre est complexe et coûteuse. Enfin, quand il s'agit d'établissements où la France apporte un appui pédagogique, cet appui doit être revu et notablement accentué. Les coûts totaux peuvent alors devenir fort élevés, en particulier si de nombreux établissements du même pays se 
proposent de préparer le baccalauréat français et si d'autres pays décident d'en faire autant.

À ce niveau, une telle décision ne peut plus appartenir à l'établissement et à la communauté scolaire seule. Elle devient politique et touche autant la France que le pays d'accueil, la France parce que c'est à elle que reviendra la prise en charge de l'examen, le pays d'accueil parce qu'il lui revient d'autoriser ou non la préparation, et donc souvent d'accorder la reconnaissance, d'un examen étranger. Ceci ne va d'ailleurs pas sans poser de problème dans le cas fréquent où l'examen local de fin d'études secondaires ne donne pas un accès direct à l'université, alors que le baccalauréat français, considéré en France comme le premier diplôme de l'enseignement supérieur, permet d'y accéder directement. Le nouveau bachelier, après une scolarité forcément plus difficile que celle de ses camarades non bilingues puisque suivie dans un système éducatif étranger, se retrouverait alors doublement défavorisé, parce que devant préparer de toutes manières des examens d'entrée à l'université qui s'appuient sur les programmes nationaux qu'il n'aurait pas suivis.

Du côté français, le problème du coût est évidemment essentiel : il s'agit de décider si l'investissement vaut la peine d'être tenté en termes de retombées d'influence prévisibles à longue échéance. Il n'est cependant pas le seul à devoir être pris en compte. Si la politique linguistique d'un pays se décide en grande partie en fonction des moyens dont ce pays dispose et qu'il décide d'y consacrer, elle doit être influencée également par des considérations moins triviales. Est-il légitime, par exemple, qu'un pays «exporte » ses examens et son système éducatif comme il le ferait d'une marchandise ordinaire? La France, en tout cas, s'y est refusé pour les établissements bilingues, même quand elle aurait pu le faire parce que certains de ses partenaires le lui demandaient.

\section{Quels manuels, quelle certification, quelle formation des enseignants?}

Qu'on écarte la solution qui consiste à adopter les programmes français et l'on n'est pas pour autant sorti de l'auberge. Dans le cas de programmes nationaux en effet, les problèmes qui se posent sont nombreux: quels manuels utiliser? Quelle validation mettre en place? Quelles adaptations envisager?

Le problème des manuels se pose très vite. Un enseignement bilingue nécessite en effet des manuels rédigés dans la langue étrangère d'enseignement. Plusieurs solutions sont possibles :

- une simple traduction des manuels existants (cas fréquent);

- l'élaboration de manuels spécifiques, solution souvent revendiquée mais coûteuse, qui pose des problèmes de fond (quelle approche didactique, quelle marge de manœuvre vis-à-vis des contenus, quels concepteurs ?) et qui n'est pas sans danger pour le pays d'accueil quand la comparaison est défavorable aux productions locales ;

- l'élaboration de documents complémentaires, solution bâtarde mais qui présente des avantages, tant au plan financier qu'à celui des susceptibilités locales.

Le problème de la validation est évidemment central. L'enseignement bilingue nécessite pour les élèves qui le pratiquent un investissement plus important que celui qui serait le leur dans le système monolingue, leur formation est plus complète que celle de leurs camarades et ils acquièrent des compétences linguistiques supérieures. Il serait donc 
légitime que cet effort et ces compétences soient reconnus. C'est encore rarement le cas. Le plus souvent l'élève bilingue passe les examens locaux comme ses camarades des établissements monolingues. Certains pays ont inauguré des formules variées. En Égypte, on peut passer cet examen local en français (choix de $80 \%$ des élèves des établissements francophones) ou en arabe, en Allemagne, l'Abitur est agrémenté d'une mention spécifique si les épreuves sont passées en français, en République tchèque et en Slovaquie existe une "Maturita bilingue " qui ne permet pas cependant l'accès à l'université, contrairement par exemple à l'examen hongrois. Si une validation officielle semble légitime du côté du pays qui a mis en place un enseignement bilingue, elle l'est aussi pour la France qui, à partir de cette année, remet une attestation spécifique à chaque élève des établissements et sections bilingues francophones ayant réussi l'examen de fin d'études secondaires. Cette attestation dispense du test linguistique nécessaire à l'entrée dans l'enseignement supérieur en France. Il s'agit aussi d'un geste symbolique vis-à-vis des partenaires étrangers destinés à les engager à valider de leur côté la compétence des élèves bilingues francophones.

L'adaptation des programmes peut ne pas sembler une urgence dans un premier temps; elle se pose pourtant souvent au bout de quelques années, au moins pour les enseignants et les familles, ne serait-ce que parce que les exigences des uns et des autres, liées à la revendication d'excellence des cursus bilingues, revendication souvent proche d'une « distinction » sociale, deviennent plus importantes.

Qu'un pays accepte une adaptation de ces programmes officiels et il lance un message fort quant à sa volonté d'ouverture aux autres langues et cultures. Cela peut prendre la forme, comme en Bulgarie, par exemple, pour les disciplines scientifiques, d'un rapprochement entre programmes français et nationaux, mettant en commun ce qui est proche et essayant d'harmoniser les différences, première forme d'un rapprochement non seulement des contenus mais des démarches didactiques et des méthodes d'enseignement.

Lorsqu'il s'agit de disciplines sensibles comme l'histoire ou la géographie, le message devient plus fort encore. Tout rapprochement dans ces domaines nécessite en effet que le pays qui s'y décide soit parfaitement serein vis-à-vis de son propre passé et de son histoire présente, ce qui n'est pas toujours le cas. C'est une des raisons pour lesquelles il est souvent plus facile d'introduire un enseignement bilingue dans les disciplines scientifiques plutôt que dans les sciences sociales. Lorsque l'enseignement de l'histoire et de la géographie se fait en français, on le couple souvent avec un enseignement de ces matières dans la langue nationale. C'est le cas dans la plupart des pays d'Amérique latine, c'est le cas aussi dans certains pays d'Europe orientale. On sent bien que l'enseignement bilingue, forcément biculturel puisque langues et cultures sont inséparables, prend toujours place au sein d'enjeux nationaux qui le dépassent largement et qui n'ont pas grand chose à voir avec le didactique ou le cognitif.

30 Même s'il n'y a pas "exportation " d'examens ou de programmes, l'enseignement bilingue pose en effet un problème d'identité culturelle qu'il convient ni de surestimer ni de sous-estimer. Il doit certes permettre de faire acquérir à des élèves un excellent niveau dans une langue autre que leur langue maternelle, mais, la langue étant un vecteur de culture, l'élève ressort forcément du système plus bousculé culturellement que l'élève ayant suivi une scolarité monolingue. Sans doute, tout enseignement inclut-il une forme de « bousculement » nécessaire puisqu'il n'y a pas d'apprentissage possible sans remise en cause du connu, sans doute le monde moderne force-t-il à des remises en cause bien plus 
traumatisantes, il n'empêche que lorsque le nombre des jeunes concernés devient important, ce «bousculement » culturel risque de devenir un fait social dont la résolution gagnerait à avoir été envisagé par les autorités éducatives dans le cadre de leur politique linguistique intérieure.

Qu'il y ait ou non adaptation des programmes, le problème de la formation des enseignants est bien évidemment central. La formation linguistique est première, en particulier pour les enseignants des disciplines autres que le français puisqu'il est exceptionnel, et peu souhaitable d'ailleurs, que l'enseignement de spécialité soit dispensé par des professeurs de langue. Il est rare en effet de trouver des enseignants suffisamment bilingues pour enseigner dans une autre langue que la leur. C'est la raison première de l'échec relatif de l'enseignement bilingue en France même. On peut bien évidemment faire appel à des enseignants natifs, mais l'opération est coûteuse, surtout si elle touche un grand nombre d'établissements, et elle n'est pas sans poser le problème de la capacité future pour le pays d'accueil d'assumer seul ses choix éducatifs.

D'autre part, cette formation linguistique, pour être efficace, doit être intensive, prolongée, répétée, et se faire en partie en France, ce qui augmente considérablement les coûts de l'enseignement bilingue, tant pour le pays d'origine des enseignants que pour la partie française. Dans la plupart des cas, et en particulier lorsqu'il y a adaptation des programmes, s'ajoute à cette formation linguistique un complément de formation pédagogique et didactique. L'enseignement bilingue en effet ne peut être un simple calque ou une simple traduction de l'enseignement traditionnel. Il prétend le plus souvent à une qualité supérieure, non seulement pour le degré de compétence linguistique mais aussi pour les acquisitions académiques et la formation de l'esprit. C'est d'ailleurs souvent cet apport supplémentaire qui est à l'origine de la motivation des familles. On touche alors, plus que dans le cas de la formation linguistique, une corde sensible pour les autorités éducatives nationales qui risquent de voir remises en cause, en particulier quand il est fait appel à des experts étrangers, les formations locales qu'elles ont mises en place. Dès qu'une réflexion sur les méthodes d'enseignement est lancée, par exemple, c'est le rapport entre maîtres et élèves qui sont posé et, par voie de conséquence, potentiellement, celui des relations entre les citoyens et les détenteurs de l'autorité. Ce type de questionnement, on le comprendra, ne gêne pas seulement les systèmes éducatifs reposant sur une tradition confucéenne...

\section{Les enjeux de l'enseignement bilingue}

Pour toutes ces raisons, l'enseignement bilingue pose toujours problème au sein d'un système éducatif et est souvent l'objet de débats politiques intenses qui pourraient se résumer en une phrase : le jeu en vaut-il la chandelle? Le «jeu », c'est l'ensemble des avantages qu'on pense tirer de la pratique d'un bilinguisme où l'autre langue est celle d'un pays étranger, parfois fort éloigné culturellement (il convient de mettre à part ici l'enseignement bilingue s'appuyant sur des langues régionales ou nationales, en Catalogne par exemple) ; la " chandelle ", c'est le risque de remise en cause d'une identité linguistique et culturelle parfois difficilement acquise, voire pas du tout. Il apparait le plus souvent que, plus cette identité est forte et plus elle plonge profondément ses racines dans l'histoire, moins ce risque est grand. langue par exemple, peut être alors, à juste titre, mal perçue. Inversement, le pays 
diffuseur peut avoir scrupule, souvent pour des raisons liées à l'histoire ou au rôle qu'il a décidé de jouer dans le monde, à aller trop loin dans cette diffusion. D'où des incompréhensions mutuelles parfois, et dans les deux sens.

Qu'un pays définisse une politique linguistique très ouverte aux autres langues, comme certains pays d'Europe centrale et orientale, et il peut trouver en face de lui des pays à la politique linguistique extérieure trop timorée à son gré, ce qui n'empêchera pas d'ailleurs le pays demandeur de mettre en place les structures d'enseignement adéquates s'il en a les moyens.

Qu'un pays, au contraire, parfois parce que son identité nationale est encore à conquérir, ou parce qu'un passé colonial reste présent à la mémoire, adopte une politique linguistique intérieure prudente et il considérera toute volonté extérieure de diffusion d'une langue étrangère comme une ingérence susceptible de mettre en danger sa propre culture ou les efforts entrepris pour unifier des langues et cultures nationales amenées à coexister. Dans certains cas, au Cambodge par exemple, c'est moins la diffusion de la langue étrangère qui pose problème que son utilisation, même partielle, comme langue d'enseignement au sein du système éducatif national, surtout quand celui-ci s'appuie sur une langue locale intégrant de manière insuffisante la terminologie et les concepts permettant un enseignement moderne. La priorité, dans ce cas, peut consister à faire acquérir aux yeux mêmes de ses utilisateurs naturels une légitimité à leur langue maternelle qui passe, d'une part par une adaptation du lexique de cette langue au monde d'aujourd'hui, et d'autre part par une politique linguistique la favorisant. Le problème ne se pose d'ailleurs pas seulement quand la langue nationale doit se défendre face à la place croissante d'une langue étrangère, mais dans de nombreuses situations de diglossie. Certains pays d'Amérique latine, à populations à majorité ou à fortes minorités non hispanophones, dont les langues, essentiellement orales jusqu'ici, pourraient devenir partiellement langues d'enseignement (Bolivie et Paraguay, par exemple) sont actuellement confrontés à ce problème.

L'enseignement bilingue, comme tout enseignement de langues étrangères d'ailleurs, se trouve donc à ce croisement entre deux politiques linguistiques qui parfois se rejoignent et parfois se séparent. L'histoire récente de l'extension de l'enseignement bilingue francophone est faite essentiellement de ce mouvement d'oscillation, qui varie d'un pays à l'autre et, pour un même pays, d'un moment à l'autre de son histoire.

Dans ce cadre, prétendre promouvoir l'enseignement bilingue en soi n'a guère de sens, sinon au nom d'une idéologie universaliste certes généreuse mais qui ne mesure pas toujours très bien les conséquences d'une telle revendication. Parler la langue de l'autre n'est hélas pas suffisant en effet pour développer l'amitié entre les peuples et nous préserver à jamais des guerres, comme on a pu le croire à une certaine époque et comme une partie de l'opinion publique continue encore à le croire. L'histoire récente montre assez que les guerres civiles et les guerres fratricides sont souvent parmi les plus cruelles et que le fait de bien se comprendre n'arrange pas forcément les choses.

39 Promouvoir l'enseignement bilingue, ce n'est donc pas se contenter de prêcher pour une meilleure communication entre les hommes, c'est en fin de compte agir sur le sens de cette communication, et donc l'orienter. C'est ainsi que la simple revendication bilingue ne prend le plus souvent en compte que les avantages individuels de la bilingualité sans voir qu'un risque existe d'arriver, non pas à l'apprentissage, de manière égalitaire, de la langue des autres, mais, dans le monde actuel, à l'installation dans beaucoup de pays d'une langue unique d'enseignement, l'anglais, qui, on le sait, tend à être de plus en plus 
utilisée comme langue de communication internationale standard, qui est perçue parfois comme ayant vocation universelle (certains parlent même de sa facilité d'apprentissage), et dont l'extension profite directement, du moins aujourd'hui, aux pays l'employant comme langue maternelle. On voit bien, dans ce contexte, comment l'idéologique peut être vite rattrapé par le politique et l'économique.

S'il est devenu banal en effet de rappeler que le monolinguisme est l'exception dans le monde et que la plupart des hommes et des femmes de cette planète sont exposés au quotidien, et depuis bien longtemps, à des situations de plurilinguisme, il est tout aussi banal de constater que les langues auxquelles sont confrontés ces hommes et ces femmes ne sont pas égales entre elles, d'abord au plan individuel, parce que le bilinguisme équilibré est une situation exceptionnelle et que, même dans ce cas, chacune des langues est utilisée et vécue par l'individu différemment de l'autre, au plan social ensuite, parce que les statuts réels de ces langues sont eux aussi inégaux, quand bien même les statuts officiels visent à une égalité apparente. Dès lors qu'il y a inégalité, apparaissent inévitablement frustrations et revendications donc situations potentielles de confrontation et de conflit. Ainsi, la coexistence de deux ou de plusieurs langues dans un champ donné d'observation, quelle que soit la taille de ce champ, risque d'entraîner des affrontements qui, lorsqu'ils se situent à un niveau individuel, déterminent des attitudes ayant inévitablement, quand elles s'additionnent, des conséquences sociales, et qui, lorsqu'ils se situent à un niveau collectif, débouchent forcément sur des enjeux de groupes. Parler de langues, on le sait bien, n'est jamais neutre et s'inscrit dans un discours plus global, évidemment politique. C'est pourquoi, dès qu'on se situe dans un cadre diglossique, et l'on sait que toute situation bilingue est diglossique, prendre position pour une langue revient à prendre position pour le groupe qui s'identifie à cette langue.

41 Sur un problème comme celui de l'enseignement bilingue, toute prise de position dépasse de beaucoup, on le voit, le didactique. Mais tout silence est aussi positionnement. Ainsi, aucun gouvernement, dès qu'il gouverne ne peut échapper à la mise en œuvre d'une politique linguistique ou, à défaut, d'une glottopolitique. Une politique linguistique en effet n'est pas toujours, est même rarement, définie en tant que telle. C'est notamment le cas de certains pays qui accueillent sur leur sol un enseignement bilingue. En France même, il existe moins une volonté que des volontés, des tendances, des aspirations, souvent confuses, éparses, à un tel enseignement, sans qu'on sache bien quelles pourraient en être les finalités si ce n'est quelque chose de vague qui se rapporte, tantôt à une future citoyenneté européenne, tantôt à des désirs parentaux, tantôt à la conscience de l'importance des langues dans le monde d'aujourd'hui, tantôt à une générosité de principe. Les autres pays ne sont en général pas plus avancés dans leur réflexion, ils le sont même souvent moins, laissant parfois ainsi au sein de leur système éducatif des brèches où il est possible de s'engouffrer.

42 C'est même le plus souvent au sein de telles brèches, de tels espaces flous, qu'un enseignement en français s'installe ou a pu s'installer dans le passé. L'acquis n'est jamais forcément définitif (on l'a vu en Syrie lors de la nationalisation des établissements de la Mission laïque française) mais il est rarement remis en cause.

Ce qui apparaît aujourd'hui c'est donc une situation relativement nouvelle. Pendant des dizaines d'années, il semblait que les établissements bilingues (lycées français inclus) ne posaient aucun problème particulier, si ce n'est celui de leur fonctionnement quotidien. Plusieurs éléments sont venus les mettre au premier plan. 

instruments prestigieux mais coûteux. En cherchant à les rendre moins chers on s'est interrogé sur leur vocation. En 1985, le colloque de Madrid avançait la problématique de l'enseignement dans les lycées français dans une perspective bilingue et biculturelle. En 1988, le colloque de Montevideo posait le problème de l'insertion des établissements français d'Amérique latine dans leur contexte local. La même année, dans son Nouveau discours sur l'universalité de la langue française, Thierry de Beaucé, futur secrétaire d'État chargé des relations culturelles internationales déclarait: «Le système des établissements français tourne en rond sans objectifs définis ni visions d'avenir ». Le $1^{\text {er }}$ janvier 1990 était créée l'Agence pour l'enseignement du français à l'étranger (AEFE).

Parallèlement, c'est en 1990 également que s'ouvraient en nombre, à la faveur de la chute du rideau de fer, des sections bilingues dans les pays d'Europe centrale et orientale. En 1992, les premières classes bilingues étaient ouvertes au Vietnam. La première filière universitaire francophone était créée la même année en Égypte. Et le mouvement s'est accéléré un peu partout depuis, installant une situation nouvelle, qui apparait comme le résultat d'une politique volontaire, tant de la part du pays diffuseur de sa langue qu'est la France que de celle des pays demandeurs, mais qui, en même temps, oblige à apporter des réponses à des questions qui ne se posaient guère jusqu'ici et dont on a vu la variété et l'ampleur.

C'est peut-être la raison pour laquelle aujourd'hui une réflexion nouvelle s'amorce, et dans toutes les directions. Si du point de vue psycholinguistique, cette réflexion est déjà ancienne et bien avancée, le contexte nouveau peut aider à revigorer la didactique des langues étrangères, mais aussi ouvrir des perspectives prometteuses aux didactiques des différentes disciplines. C'est peut-être cependant la sociolinguistique qui pourrait tirer de la situation actuelle le plus de profit par le spectacle que nous donnent des politiques linguistiques en action. Ce spectacle risque d'être d'autant plus intéressant que nous en sommes tous potentiellement les acteurs.

\section{INDEX}

\section{Index géographique : France}

Mots-clés : enseignement bilingue, formation des enseignants, politique linguistique, section bilingue, enseignement du français, FLE

\section{AUTEUR}

\section{GUY TRÉZEUX}

Direction générale des relations culturelles, scientifiques et techniques, ministère des affaires étrangères, Paris, France 